\title{
Spectrum Auction Framework for Access Allocation in Cognitive Radio Networks
}

\author{
Gaurav S. Kasbekar \\ Department of Electrical and Systems \\ Engineering \\ University of Pennsylvania \\ kgaurav@seas.upenn.edu
}

\author{
Saswati Sarkar \\ Department of Electrical and Systems \\ Engineering \\ University of Pennsylvania \\ swati@seas.upenn.edu
}

\begin{abstract}
Cognitive radio networks are emerging as a promising technology for the efficient use of radio spectrum. In these networks, there are two categories of networks on different channels: primary networks and secondary networks. A primary network on a channel has prioritized access to the channel and secondary networks can use the channel when the primary network is not using it. The access allocation problem is to select the primary and secondary networks on each channel. We develop an auction-based framework that allows networks to bid for primary and secondary access based on their utilities and traffic demands, and uses the bids to solve the access allocation problem. We develop algorithms for the access allocation problem and show how they can be used either to maximize the auctioneer's revenue given the bids, or to maximize the social welfare of the bidding networks, while enforcing incentive compatibility. We first consider the case when the bids of a network depend on which other networks it will share channels with. When there can be only one secondary network on a channel, we design an optimal polynomial-time algorithm for the access allocation problem based on reduction to a maximum matching problem in weighted graphs. When there can be two or more secondary networks on a channel, we show that the optimal access allocation problem is NP-Complete. Next, we consider the case when the bids of a network are independent of which other networks it will share channels with. We design a polynomial-time dynamic programming algorithm to optimally solve the access allocation problem when the number of possible cardinalities of the set of secondary networks on a channel is upper-bounded. Finally, we design a polynomial-time algorithm which approximates the access allocation problem within a factor of 2 when the above upper bound does not exist.
\end{abstract}

\section{Categories and Subject Descriptors}

C.2 [Computer-Communication Networks]: Wireless Communication

Permission to make digital or hard copies of all or part of this work for personal or classroom use is granted without fee provided that copies are not made or distributed for profit or commercial advantage and that copies bear this notice and the full citation on the first page. To copy otherwise, to republish, to post on servers or to redistribute to lists, requires prior specific permission and/or a fee.

MobiHoc'09, May 18-21, 2009, New Orleans, Louisiana, USA.

Copyright 2009 ACM 978-1-60558-531-4/09/05 ...\$5.00.

\section{General Terms}

Algorithms, Design, Economics, Theory

\section{Keywords}

Cognitive Radio Networks, Spectrum Auctions, Algorithms

\section{INTRODUCTION}

With the proliferation of different wireless network technologies like cellular networks, Wireless Local Area Networks, Wireless Meteropolitan Area networks etc., demand for radio spectrum is increasing. Currently, spectrum is regulated by a government agency like the Federal Communications Commission (FCC) and it allocates spectrum by assigning exclusive licenses to users to operate their networks in different geographical regions.

There is a widespread belief that radio spectrum is becoming increasingly crowded. However, spectrum measurements indicate that the allocated spectrum is under-utilized, i.e. at any given time and location, much of the spectrum is unused [2]. Cognitive radio networks are emerging as a promising solution to this dilemma. In these networks, there are two levels of networks on a channel- primary networks and secondary networks. A primary or secondary network is a network of multiple wireless devices, which we call primary and secondary nodes respectively. A primary node has priortized access to the channel, i.e. it can transmit on the channel regardless of the transmissions of secondary nodes. On the other hand, a secondary node can transmit on the channel provided primary nodes are not transmitting. So whenever a secondary node wants to transmit on the channel, it senses the channel to check for ongoing transmissions. It initiates a transmission only if a primary node is not transmitting. Moreover, if a secondary node is transmitting and a primary node wishes to transmit, then the secondary node suspends its transmission until after the primary node finishes its transmission. Cognitive radio technology [4] allows secondary nodes to detect which channel is not being used by primary nodes, share this channel with other secondary nodes and vacate the channel when a primary node is detected. Surveys on cognitive radio networks can be found in [1] and [4].

An important question faced by a spectrum regulator is how to allocate the rights to be primary and secondary networks on its channels. Different networks may attach different value to being primary and secondary. A network may wish to mainly transmit delay-sensitive traffic like voice or video. Such a network will attach a high value to the 
rights to be primary. On the other hand, a network may be mainly interested in transmitting delay-insensitive traffic like email or file transfer. Such a network would not need primary rights and would prefer secondary rights since the latter would be priced lower than the former. Also, a network whose traffic is a mixture of delay-sensitive and delay-insensitive traffic would want primary rights on some channels and secondary rights on some channels.

Auctions are suitable for selling the rights to be primary and secondary on the channels. Since the regulator need not know the values that bidders attach to primary and secondary rights, auctions provide a mechanism for the regulator to get a higher revenue than that obtainable through static pricing [9]. Auctions are also beneficial for the bidders since in general they assign goods to the bidders who value them most [9]. FCC has been conducting spectrum auctions since 1994 to allocate licenses for radio spectrum [3] (however, so far, no auctions have been conducted for cognitive radio networks).

Spectrum auctions have been studied in [9], [6], [7], [8]. We now explain how our work differs from previous work. In some of the existing work on spectrum auctions [9], [8], each channel is assigned to a single network, i.e. there is no notion of primary and secondary networks on a single channel. We consider the case when there is a primary network and one or more secondary networks on each channel. Now, there are two possibilities [10] for allocating secondary rights on the channels. In one possibility, the regulator allocates channels to primary networks and the primary networks independently allocate unused portions on their channels to the secondary networks. Auctions have been designed for this scenario in [6] and [7]. In the other possibility, the regulator allocates the rights to be the primary and secondary networks on the channels in a single allocation [10]. To the best of our knowledge, no work has been done in designing auctions for this scenario. In this paper, we develop a comprehensive auction framework using which a regulator can simultaneously allocate the rights to be primary and secondary on the channels. This scenario may be more desirable than the first possibility above in certain cases. For example, it gives a greater degree of control over the allocation to the regulator than in the case when primary networks allocate unused portions on their channels to the secondary networks.

We consider a scenario in which the regulator conducts an auction to sell the rights to be primary and secondary networks on a set of channels. Networks can bid for these rights based on their utilities and traffic demands. The regulator uses these bids to solve the access allocation problem, i.e. the problem of deciding which networks will be the primary and secondary networks on each channel. The goal of the regulator may be either to maximize its revenue or to maximize the social welfare of the bidding networks. Now, networks can have utilities or valuations that are functions of the number of channels on which they get primary and secondary rights, on how many and which other networks they share these channels with etc. The number of valuations of a network may be large and an exponential amount of space may be required to express a bid for each valuation. So we design bidding languages, that is, compact formats for networks to express bids for their valuations. For different bidding languages, we design algorithms for the access allocation problem.
The paper is organized as follows. We describe the system model in Section 2. In Section 3, we describe how the bidding languages and algorithms that we design in the paper can be used to maximize the auctioneer's revenue or to maximize social welfare. In Section 4, we describe a model in which the bids of a network depend on which other networks it will share channels with. In Section 4.1, we design an optimal algorithm for the access allocation problem for a simple case with only one secondary network on each channel. We show the intractability (NP-Competeness of the access allocation problem or exponential size of bids) of the extensions of this simple case in Section 4.2. In Section 5, we consider the case in which the bids of a network are independent of which networks it will share channels with and provide an optimal polynomial-time algorithm for the access allocation problem when the number of cardinalities of the set of secondary networks on a channel is upper-bounded. In Section 6, we describe a bidding language that can be used for the independent bids case when the above bound does not exist and provide a greedy 2-approximation algorithm for the access allocation problem.

Due to space constraints, we omit the proofs of several results and outline the proofs for some others.

\section{SYSTEM MODEL}

We consider a scenario in which there are $M$ identical orthogonal channels in a region. A regulator conducts an auction to sell the rights to be the primary and secondary networks on the channels. $N$ bidders participate in the auction. Each bidder is an independent network of multiple wireless nodes. Each bidding network submits bids to the regulator and based on the bids, the latter allocates the rights to be the primary and secondary networks on the channels.

A primary network on a channel must have priortized access to the channel. If two or more independent networks were to be the primary networks on a single channel, then the access of each one of them would be constrained by the transmissions of the other primary networks, which would transmit at the same priority level. To avoid this, we assume that there is exactly one primary network on each channel. However, we allow multiple networks to have secondary rights on a channel.

We assume that all the secondary networks on a channel have equal rights on the channel. This is because complicated multiple access protocols [5] would be required to grant access at different priority levels to different secondary networks on a channel (with all of them getting lower priority access than the primary network). On the other hand, simple multiple access protocols would suffice if all secondary networks have equal rights on the channel.

Now, since a primary network has priortized access on a channel, the average delay of its traffic is low. On the other hand, the average delay of a secondary network's traffic is high. Hence, primary rights (respectively secondary rights) are suitable for communicating delay-sensitive (respectively delay-insensitive) traffic. We assume that each network has two kinds of traffic: (a) delay-sensitive traffic like voice, video etc. and (b) delay-insensitive or elastic traffic like email, file-transfer etc. A network uses its primary rights to transmit its delay-sensitive traffic and its secondary rights to transmit its elastic traffic.

We allow a single network $i$ to be both the primary network and one of the secondary networks on a channel. In this 
case, we assume that it transmits its delay-sensitive traffic as a primary network, i.e. with high-priority and elastic traffic as a secondary network, i.e. when it has no delay-sensitive traffic to transmit. Also, the other secondary networks on the channel can transmit whenever network $i$ is not transmitting its delay-sensitive traffic.

Let $K$ be the set of all possible ways in which the $M$ channels can be allocated to the $N$ bidders. For example, consider the simple case in which $M=3, N=9$ and there can be at most four secondary networks on a channel. An example of an allocation of the channels is one in which network 1 becomes the primary network on channels 1 and 2, network 2 becomes primary on channel 3, network 3 becomes the sole secondary network on channel 1 , networks 4 and 5 become secondary networks on channel 2, networks 1, 4, 6 and 7 become secondary networks on channel 3 and networks 8 and 9 do not become primary or secondary networks on any channel.

Let $x_{i}(k)$ be network $i$ 's valuation or utility from the channel allocation $k \in K$, i.e., the value that it conjectures or expects to derive from the allocation $k$. Note that since network $i$ will share channels with other networks in the allocation $k$, the actual utility that network $i$ will derive from an allocation $k$ depends on the transmission patterns of the other networks that are not completely known to network $i$. Hence, each network $i$ bids for access based on its conjectures about its actual utility. Henceforth, we use the terms valuation or utility for $x_{i}(k)$, but they should be understood to mean the conjectured utility or valuation of network $i$ for the channel allocation $k$.

The valuations $x_{i}($.$) of network i$ for the allocations in $K$ depend on its traffic demands, i.e. the volumes of delaysensitive and elastic traffic that it wants to transmit. Now, for given traffic demands, the valuation of a network $i$ for a channel allocation $k \in K$ may depend upon the number of channels on which network $i$ has primary and secondary rights in the allocation $k$, how many and which other networks have rights on each of the channels on which network $i$ has primary or secondary rights etc. Note that network $i$ may have the same valuation for different allocations $k \in K$.

Network $i$ 's net utility is of the form:

$$
u_{i}\left(k, \tau_{i}, x_{i}\right)=x_{i}(k)-\tau_{i}
$$

where $\tau_{i}$ is the payment that network $i$ makes to the auctioneer. The auctioneer determines the channel allocation and the payment $\tau_{i}$ that each network $i$ makes to the auctioneer. The social welfare of an allocation $k$ is defined to be the quantity:

$$
\sum_{i=1}^{N} x_{i}(k)
$$

Thus, the social welfare is the sum of utilities of all bidders from the allocation $k$.

Now, there could be two goals for designing the auction: revenue maximization and maximizing social welfare. In the first goal, based on its valuations, each network submits a set of bids to the auctioneer. Let $z_{i}(k)$ be the bid of network $i$ for the allocation $k \in K$, i.e. the amount of money it is willing to pay if the allocation $k \in K$ is chosen. Let $k^{*}$ be the channel allocation that maximizes the revenue of the auctioneer, given the bids $z_{i}($.$) for bidders 1, \ldots, N$. That is, $k^{*}$ satisfies:

$$
\sum_{i=1}^{N} z_{i}\left(k^{*}\right) \geq \sum_{i=1}^{N} z_{i}(k) \quad \forall k \in K
$$

As we will see in Section 3, when $z_{i}($.$) are not the bids of the$ networks, but have a different interpretation, the channel allocation that maximizes the social welfare of the $N$ networks can be found by finding the $k^{*}$ satisfying the above equation. The access allocation problem is to determine the channel allocation $k^{*}$ satisfying (2). Depending on the interpretation of $z_{i}($.$) , this allocation k^{*}$ either maximizes the auctioneer's revenue or the social welfare of the $N$ networks.

Now, the set $K$ of possible channel allocations may be exponential in size. As noted earlier, a bidder $i$ may have the same valuation for two or more allocations in $K$ and hence it need not specify a bid for each $k \in K$. The total number of different valuations of network $i$ may still be exponential. However, it is not feasible to communicate a bid for each valuation in this large set. So we introduce bidding languages for the auction models that we consider. A bidding language [11] is a format to compactly encode the bid information of a bidder. When there are an exponential number of valuations, a bidding language expresses the bids approximately, not exactly.

\section{SOLUTION FRAMEWORK}

As stated earlier, an auction could be designed for two different objectives. In our context, the first objective is to choose the channel allocation that maximizes the regulator's revenue for a given set of bids of the bidders. This can be done by choosing the allocation $k^{*}$ satisfying (2) when $z_{i}(k)$ is the bid of network $i$ for the channel allocation $k$.

The second possible objective for the auction could be to achieve efficiency, that is, to choose the allocation that maximizes social welfare. To this end, each bidder is asked to declare its valuation function $x_{i}($.$) . With an abuse of nota-$ tion, let $z_{i}(k)$ denote the declared valuation of network $i$ for the allocation $k$, which may be different from $x_{i}(k)$ if bidder $i$ believes that falsely declaring its valuations will improve its net utility. Truth-telling is said to be a weakly-dominant strategy [17] for network $i$, if for any possible declarations of networks other than $i$, the net utility of network $i$ is maximized when it sets $z_{i}(k)=x_{i}(k) \forall k \in K$. It follows from the revelation principle [17] that to maximize social welfare, it is sufficient to consider mechanisms in which the payments $\tau_{i}$ are chosen such that for each bidder $i$, truth-telling is a weakly dominant strategy. Such a mechanism is called incentive compatible.

To date, the Vickrey-Clarke-Groves (VCG) mechanism [17] is the only known general incentive compatible mechanism that can be used to maximize social welfare. Under this mechanism, given the declared valuation functions $z_{i}($.$) of$ the bidders, the allocation $k^{*}$ satisfying (2) is chosen and the payments are chosen so as to enforce incentive compatibility [17]. It can be shown that the VCG mechanism can be implemented by running an algorithm for the access allocation problem (in equation $(2))(N+1)$ times- once with all bidders and once each for the set of bidders $\{1, \ldots, N\} \backslash i$ for $i=1, \ldots, N$.

Now, in general, the set of different valuations of a bidding network is exponential in size. First we consider the special case when the number of different valuations of each bidding 
network is of poynomial space complexity (but $K$ can still be exponential in size). Even in this case, it is sometimes computationally intractable to devise an algorithm to find the optimal allocation $k^{*}$ satisfying (2), possibly because this is NP-hard, but instead an approximation algorithm for the access allocation problem can be devised. In this case, the VCG mechanism cannot be used to enforce incentive compatibility. To address this problem, Nisan and Ronen [19] devised the second-chance mechanism which enforces incentive compatibility under the assumption that there is a limit on the computational resources of each bidder. Moreover, the social welfare attained by the second-chance mechanism is at least as good as the social welfare of the approximation algorithm used for the access allocation problem.

Now, in some cases, the set of valuations of a bidder takes an exponential amount of space and hence bidders have to use incomplete bidding languages (see Section 2) to convey their valuations. In this case as well, the VCG mechanism cannot be used to enforce incentive compatibility. As a solution to this problem, Ronen [20] devised the extended secondchance mechanism that, under reasonable assumptions [20], can be used to enforce incentive compatibility and achieve a social welfare at least as good as that of the approximation algorithm used for the access allocation problem.

In this paper, we propose several spectrum auction models and design bidding languages and algorithms for the access allocation problem. These can be used for the objective of maximizing the revenue of the auctioneer or for maximizing the social welfare of the bidders in conjunction with the VCG, second-chance or extended second-chance mechanism, as appropriate.

For notational convenience, throughout the paper, we assume that $z_{i}($.$) are the bids expressed by bidder i$ and view the access allocation problem as the problem of maximizing the revenue of the auctioneer. However, our framework applies without change to the problem of maximizing social welfare.

\section{AUCTION WITH DEPENDENT BIDS}

A primary or secondary network on a channel shares the channel with other networks and hence its actual utility from the channel depends on the transmissions of those networks. A network may have some knowledge or beliefs about the typical transmission patterns of the other bidding networks. For example, the agency owning the network may conduct a survey on the typical transmission patterns of the other networks in its region or, if auctions are periodically conducted to allocate spectrum in the region, the agency may gain this knowledge about the networks with whom it shared channels previously. Thus, the conjectured utilities and hence the bids of a network would depend on which networks it will share different channels with.

\subsection{Basic Model}

In the basic model with dependent bids, we consider the model described in Section 2 with the following additional assumptions.

Assumption 1. There is only one secondary network on each channel.

ASSUMPTION 2. Each network can be either the primary or the secondary network on only one channel.
We explore the effect of relaxing either of these assumptions in Section 4.2. We assume that $N \geq 2 M$, so that all $M$ channels can be allocated.

A secondary network on a channel can use the channel whenever the primary network is not using it. So the throughput and delay of the secondary network on the channel depends on the channel usage behavior of the primary on the channel, i.e. on the rate of its transmissions on the channel and how these transmissions are spread over time. On the other hand, the primary network on a channel has priortized access to the channel. That is, when the secondary network wants to transmit on the channel, it senses the channel and can transmit only if it finds that the primary network is not transmitting. However, due to the imperfect nature of sensing, the secondary network will sometimes transmit while the primary network is transmitting, resulting in a collision. Hence the primary network's utility depends on the channel usage behavior of the secondary network on the channel. Thus, the actual utility of a primary or secondary network depends on which network it shares a channel with. As explained above, a network may in general have certain beliefs about the channel usage behavior of other networks and hence may wish to express bids dependent on the network with whom it shares the channel. To model this, let

$$
z_{i}^{p}(j), j \in\{1, \ldots, N\} \backslash\{i\}
$$

be the bid of network $i$ for the case when it is the primary network on a channel and network $j$ is the secondary network on the channel. Similarly, let

$$
z_{i}^{s}(j), j \in\{1, \ldots, N\} \backslash\{i\}
$$

be the bid of network $i$ for the case when it is the secondary network on a channel and network $i$ is the primary network.

Let

$$
k=\left\{\left(i_{1}, j_{1}\right), \ldots,\left(i_{M}, j_{M}\right)\right\}
$$

be an allocation of the $M$ channels to a set of networks. $k$ is a set of $M$ orderered pairs $\left(i_{t}, j_{t}\right)$ such that network $i_{t}$ is the primary network on channel $t$ and network $j_{t}$ is the secondary network on channel $t$. Note that the revenue of the allocation $k$ is:

$$
\sum_{t=1}^{M}\left(z_{i_{t}}^{p}\left(j_{t}\right)+z_{j_{t}}^{s}\left(i_{t}\right)\right)
$$

We describe an algorithm for determining $k^{*}$, the allocation that maximizes the revenue, by reduction to a maximum weight matching problem in a graph. Let $G$ be a weighted undirected graph with $N$ nodes, one node corresponding to each network. $G$ is a complete graph, i.e. between every pair of nodes, there is an edge. Let the weight of the edge joining nodes $i$ and $j$ be

$$
w_{i j}=\max \left(z_{i}^{p}(j)+z_{j}^{s}(i), z_{j}^{p}(i)+z_{i}^{s}(j)\right)
$$

Note that the weights are nonnegative real numbers. The interpretation of the weights $w_{i j}$ is as follows. If network $i$ (respectively network $j$ ) is the primary network on a channel and network $j$ (respectively network $i$ ) is the secondary network, then the sum of the amounts paid by networks $i$ and $j$ is $z_{i}^{p}(j)+z_{j}^{s}(i)$ (respectively, $z_{i}^{s}(j)+z_{j}^{p}(i)$ ). So $w_{i j}$, the greater of these two quantities, is the maximum sum of payments of networks $i$ and $j$ if they are the two networks on the same channel. 
A matching $\mathcal{M}$ in a graph is defined to be a subset of the edges such that no two edges in the subset share a common node. The weight of a matching is the sum of the weights of its edges.

The following algorithm finds the channel allocation $k^{*}$ that maximizes the revenue:

STEP1: In graph $G$, find a matching $\mathcal{M}_{M}^{*}$ of maximum weight among matchings with exactly $M$ edges (we say how later).

STEP2: Let $e_{1}, \ldots, e_{M}$ be the $M$ edges in the matching $\mathcal{M}_{M}^{*}$. Let $e_{t}^{1}$ and $e_{t}^{2}$ be the two endpoints of edge $e_{t}$. The allocation $k^{*}$ is chosen such that for $t=1, \ldots, M$, networks $e_{t}^{1}$ and $e_{t}^{2}$ become the two networks (primary and secondary) on channel $t$. If

$$
z_{e_{t}^{1}}^{p}\left(e_{t}^{2}\right)+z_{e_{t}^{2}}^{s}\left(e_{t}^{1}\right) \geq z_{e_{t}^{2}}^{p}\left(e_{t}^{1}\right)+z_{e_{t}^{1}}^{s}\left(e_{t}^{2}\right)
$$

then network $e_{t}^{1}$ becomes the primary network on channel $t$ and network $e_{t}^{2}$ becomes the secondary network, otherwise network $e_{t}^{2}$ becomes the primary network on channel $t$ and network $e_{t}^{1}$ becomes the secondary network.

THEOREM 1. The allocation $k^{*}$ found from the matching $\mathcal{M}_{M}^{*}$ in the above algorithm is the one that maximizes the revenue.

Proof. (Outline) There is a many-to-one correspondence between the set of channel allocations and the set of matchings with exactly $M$ edges. (It is many-to-one since the allocations obtained from any allocation by swapping the roles of the primary and secondary networks on one or more channels correspond to the same matching). From the interpretation of the weight of an edge given above, it follows that the weight of a matching $\mathcal{M}_{M}$ has the maximum revenue among the revenues of the channel allocations that correspond to it. Moreover, Step 2 of the above algorithm selects the primary network on each channel from the two networks on the channel so as to maximize the sum of payments of the two networks. Finally, since $\mathcal{M}_{M}^{*}$ is the maximum weight matching among all matchings with exactly $M$ edges, we get the desired result that the channel allocation $k^{*}$ obtained from the matching $\mathcal{M}_{M}^{*}$ is the one that maximizes the revenue.

Now, it remains to show how to find the matching $\mathcal{M}_{M}^{*}$. Edmonds [13] gave a polynomial-time algorithm for finding the maximum weight matching (with any number of edges) in a graph. However, we are interested in a maximum weight matching among matchings with $M$ edges, which cannot be directly obtained by Edmonds' algorithm. It can be obtained in $O\left(M^{4}+M^{2} N^{2}\right)$ time using White's modification [14], [15] to the algorithm.

\subsection{Intractability of Extensions}

We now explore the effect of relaxing either one of Assumptions 1 and 2. Suppose Assumption 1 is relaxed and Assumption 2 is retained. That is, we assume that each network can be the primary or a secondary network on only one channel. However, there can be multiple secondary networks on a channel. We show that even if there are two secondary networks on a channel, the problem of finding a channel allocation that maximizes the revenue is NP-Complete.

Suppose there is one primary network and $r-1$ secondary networks on each channel. Let $z_{i}^{p}\left(j_{1}, \ldots, j_{r-1}\right)$ be the bid of network $i$ for the case in which it is primary on a channel and networks $j_{1}, \ldots, j_{r-1}$ are secondary. Let $z_{j_{1}}^{s}\left(i, j_{2}, \ldots, j_{r-1}\right)$ be the bid of network $j_{1}$ for the case in which network $i$ is the primary and networks $j_{1}, \ldots, j_{r-1}$ are the secondary networks. We now define the $r$-Network Dependent Bid Access Allocation Problem ( $r$-NDBAA).

Definition 1 (The $r$-NDBAA Problem). Suppose $M$ channels are to be allocated to $N$ bidders such that on each channel, one network is primary and $r-1$ networks are secondary, where $r$ is a fixed positive integer. Each bidder can be a primary or secondary network on at most one channel and the bids of networks are as given above. Find the allocation that maximizes the revenue.

The decision version of $r$-NDBAA is as follows: given a bound $D$, is there a channel allocation such that the revenue under the allocation is at least $D$ ? We next show that (the decision version of) 3 -NDBAA is NP-Complete.

\section{TheOrem 2. 3-NDBAA is NP-Complete.}

Proof. (Outline) Given an allocation of the $M$ channels, we can verify in polynomial time whether the revenue under the allocation is at least $D$. This shows that 3 -NDBAA is in the class NP.

Next, we show that the 3-Dimensional Matching problem (3DM), which is known to be NP-complete [18], is polynomialtime reducible to 3 -NDBAA, i.e. $3 \mathrm{DM} \leq{ }_{p} 3$-NDBAA. An instance of $3 \mathrm{DM}$ is as follows [18]: Given disjoint sets $A, B$, $C$ of $m$ elements each and a set $T$ of ordered triples of the form $(a, b, c)$, where $a \in A, b \in B$ and $c \in C$, do there exist a set of $m$ triples in $T$ so that each element of $A \cup B \cup C$ is contained in exactly one of these triples?

From this instance of 3DM, we construct an instance of 3-NDBAA as follows. Let there be $M=m$ channels and $3 m$ networks- one network corresponding to each element of $A \cup B \cup C$. For every set $\{i, j, l\}$ of three networks such that $(i, j, l)$ (or one of its permutations $(j, l, i),(l, j, i)$ etc.) is a triple in $T$, define all of the following bids to be equal to $\frac{1}{3}: z_{i}^{p}(j, l), z_{j}^{p}(i, l), z_{l}^{p}(i, j), z_{i}^{s}(j, l), z_{i}^{s}(l, j), z_{j}^{s}(i, l), z_{j}^{s}(l, i)$, $z_{l}^{s}(i, j), z_{l}^{s}(j, i)$. For every set $\{i, j, l\}$ of three networks such that no permutation of $(i, j, l)$ is a triple in $T$, let all of the above bids be equal to $\frac{1}{6}$. In this 3 -NDBAA problem, we ask: is there a channel allocation of the $m$ channels with revenue of at least $D=m$ ? It can be shown that the answer is yes if and only if the answer in the original 3DM problem is yes. This shows that $3 \mathrm{DM} \leq_{p} 3$-NDBAA and hence that 3 -NDBAA is NP-Complete.

By an analogous reduction from $r$-Dimensional Matching, it can be shown that $r$-NDBAA is NP-Complete for fixed $r>3$. Note that for $r>3, r$-Dimensional Matching is NP-Complete, which follows from a trivial reduction from 3-Dimensional Matching. Moreover, if $r$ is unbounded, then each bidder $i$ would have to submit an exponential number of bids $z_{i}^{p}\left(j_{1}, \ldots, j_{r-1}\right)$ and $z_{i}^{s}\left(j_{1}, \ldots, j_{r-1}\right)$. Also, we assumed that exactly $r-1$ networks are the secondary networks on a channel. If different numbers of networks can be the secondary networks, then each network would have to submit an even greater number of bids.

Now, suppose we relax Assumption 2 and retain Assumption 1. Then each network can become a primary or secondary network on up to $M$ channels. As explained above, the utility of a network from the primary or secondary rights 
on a given channel depends upon the channel usage behavior of the network it shares the channel with. However, the channel usage behavior of this network on the channel may in turn depend upon the number of channels on which it has primary and secondary rights and the channel usage behavior of the networks it shares those channels with and so on. Thus, in general, the utility of a network may depend upon which networks are the primary and secondary networks on each channel. The number of possible ways of choosing the primary and secondary networks on the $M$ channels is clearly exponential. Thus, relaxing Assumption 2 in the auction with dependent bids would require a network to express an exponential number of bids, which is infeasible.

\section{AUCTION WITH INDEPENDENT BIDS}

In Section 4, we noted that when networks have some knowledge of the channel usage behavior of other networks, they would like to express bids dependent on which networks they will share channels with. However, it is quite possible in some scenarios that networks have no knowledge of the channel usage behavior of the other bidding networks. In this case, their conjectures about the utility that they will actually get from a channel allocation would be based only on the number of channels on which they will get primary and secondary rights and the number of other networks they will share these channels with in the allocation and would be independent of which other networks they will share channels with. Thus, they would submit bids, based on these conjectured utilities, that are independent of which networks share different channels with them.

Moreover, in Section 4.2, we showed that bids of exponential size are needed in the auction with dependent bids when Assumptions 1 and 2 are relaxed. This motivates the idea that even when networks have some knowledge of the channel usage behavior of the other networks, we can obtain a compact bidding language, that is, a means for networks to approximately convey their bids, by imposing the restriction that the bids of a network be independent of which other networks it shares different channels with. We study the auction resulting from imposing this restriction in this section.

We describe the model in Section 5.1 and provide an optimal dynamic-programming algorithm for the access allocation problem in Section 5.2.

\subsection{Model}

Consider the model in Section 2 with the following additions. On each channel, one network can be the primary network and $m_{1}, m_{2}, \ldots, m_{(n-1)}$ or $m_{n}$ networks can be the secondary networks, where $1 \leq m_{1}<m_{2}<\ldots<m_{n}$. Note that $n$ is the number of possible cardinalities of the set of secondary networks on a channel.

When the results of the auction are declared, let $n_{i, 0}$ be the number of channels on which bidder $i$ is the primary network. Let $n_{i, j}, j=1, \ldots, n$ be the number of channels on which bidder $i$ is a secondary network along with $m_{j}-1$ other secondary networks.

Suppose there are $m_{j}$ secondary networks on a channel. Recall from Section 2 that each of these $m_{j}$ secondary networks have equal rights on the channel. The share of each of these networks in the secondary rights on the channel is called a secondary part of type $j$. Also, the channel is said to be divided into $m_{j}$ secondary parts of type $j$. Similarly, since exactly one network becomes a primary network on a channel, if a network is the primary network on $l$ channels, we say that it is allocated $l$ primary parts. Also, we refer to the throughput received by a network as a secondary network as its secondary throughput.

In general, network $i$ 's utility may depend not only on the total expected secondary throughput that it gets, but also on the distribution of this secondary throughput over the $M$ channels. For example, it may get the same expected secondary throughput if (a) it is the secondary network on two channels with one other secondary network on each and (b) if it is the sole secondary network on one channel. But it may prefer one of these scenarios over the other. This is because a network has to sense different channels on which it has secondary rights for ongoing transmissions and also communicate on them. There may be costs due to delays for switching the antennas of the network's nodes between different channels. To take into account this possibility, in this section, we assume that the utility of network $i$ depends not just on the expected secondary throughput (and the number of primary parts) it receives, but on the vector $\left(n_{i, 0}, n_{i, 1}, \ldots, n_{i, n}\right)$. We allow bidder $i$ to submit bids as a function of this vector.

Each bidder $i$ submits the following bid vector to the auctioneer:

$$
\begin{array}{r}
\left\{z_{i}\left(n_{i, 0}, n_{i, 1}, \ldots n_{i, n}\right): 0 \leq n_{i, 0}, n_{i, 1}, \ldots n_{i, n} \leq M\right. \\
\left.n_{i, 1}+n_{i, 2}+\ldots+n_{i, n} \leq M ; n_{i, j} \text { integer, } j=0,1, \ldots n\right\}
\end{array}
$$

where $z_{i}\left(n_{i, 0}, n_{i, 1}, \ldots n_{i, n}\right)$ is network $i$ 's bid for becoming the primary network on $n_{i, 0}$ channels and becoming a secondary network on $n_{i, j}$ channels along with $m_{j}-1$ other secondary networks, for $j=1,2, \ldots n$.

The following result can be easily proved.

LEMMA 1. The size of the bid vector submitted by each network is $O\left(M^{n+1}\right)$.

We say that an allocation $\left\{n_{i, j}: i=1, \ldots, N ; j=0, \ldots, n\right\}$ is feasible if it is possible to assign to networks, the rights to be primary and secondary on each of the $M$ channels such that network $i, i=1, \ldots, N$ is allocated $n_{i, 0}$ primary parts and $n_{i, j}$ secondary parts of type $j$ for $j=1, \ldots, n$. The following lemma describes necessary and sufficient conditions for an allocation to be feasible.

Lemma 2. An allocation $\left\{n_{i, j}: i=1, \ldots, N ; j=0, \ldots, n\right\}$ is feasible if and only if $n_{i, 0}, n_{i, 1} \ldots n_{i, n}$ for $i=1, \ldots, N$ are integers such that for some nonnegative integers $M_{j}, j=$ $1, \ldots n$ satisfying $M_{1}+\ldots+M_{n}=M$ :

$$
\begin{array}{r}
0 \leq n_{i, 0} \leq M, i=1, \ldots, N \\
\sum_{i=1}^{N} n_{i, 0}=M \\
0 \leq n_{i, j} \leq M_{j}, i=1, \ldots, N ; j=1, \ldots, n \\
\sum_{i=1}^{N} n_{i, j}=m_{j} M_{j}, j=1, \ldots, n
\end{array}
$$

Note that the integer $M_{j}$ in the above lemma corresponds to the number of channels that are divided into $m_{j}$ secondary parts of type $j$. We assume that the number of bidders is at least $m_{1}$ so that a feasible allocation exists. 
From a feasible allocation $\left\{n_{i, j}: i=1, \ldots, N ; j=0, \ldots, n\right\}$, it is easy to construct a consistent specification of the primary and secondary networks on each channel. Hence, the access allocation problem reduces to finding the feasible allocation $\left\{n_{i, j}: i=1, \ldots, N ; j=0, \ldots, n\right\}$ that maximizes the auctioneer's revenue given the submitted bid vectors $z_{i}($.$) .$

\subsection{Algorithm to find the Optimal Feasible Allocation}

In this section, we present an exact algorithm for finding the feasible allocation that maximizes the auctioneer's revenue. The algorithm is polynomial-time when $n$, the number of possible cardinalities of the set of secondary networks on a channel, is fixed (and $m_{n}$ is allowed to grow with the problem size). This special case can be useful in practice because even with small $n$, flexibility in channel allocation can be achieved by choosing $m_{1}, \ldots, m_{n}$ judiciously. For example, with $n=3$, we can choose $m_{1}=1, m_{2}=4$ and $m_{3}=8$. In this case, large chunks of secondary throughput can be allocated to a network by having it the sole secondary network on several channels and small chunks can be allocated to networks by having 4 or 8 networks share a channel.

A dynamic programming algorithm is given in [12] and [11] for the winner determination problem in a combinatorial auction with multiple units of a fixed number of different types of objects. We generalize the algorithm in [12], [11] in two directions: (a) the objects in a combinatorial auction are indivisible, whereas we need to decide into how many secondary parts to divide each channel and (b) in our auction, the allocation has to be feasible according to the conditions in Lemma 2.

Due to space constraints, we only outline our algorithm without giving details. Given the bids $z_{i}($.$) , our goal is to$ find the feasible allocation $\left\{n_{i, j}: i=1, \ldots N ; j=0, \ldots, n\right\}$ which maximizes revenue. Fix $M_{1}, \ldots, M_{n}$ satisfying $M_{1}+$ $\ldots+M_{n}=M$ such that $M_{j}$ channels are divided into $m_{j}$ secondary parts of type $j$, for $j=1, \ldots, n$. For these fixed values, let $T\left(j_{0}, j_{1}, \ldots j_{n}, i\right)$ denote the maximum possible revenue from all participating networks when $j_{0}$ primary parts and $j_{t}$ secondary parts of type $t, t=1, \ldots, n$, are to be allocated and networks $1, \ldots, i$ are participating in the auction. Thus, $T\left(M, m_{1} M_{1}, \ldots m_{n} M_{n}, N\right)$ is the maximum revenue from all $N$ networks when $M_{j}$ channels are divided into $m_{j}$ secondary parts of type $j$, for $j=1, \ldots, n$ and can be found using the following dynamic programming algorithm.

\section{Initialization}

$$
\begin{aligned}
T\left(j_{0}, j_{1}, \ldots j_{n}, 1\right)= & z_{1}\left(j_{0}, j_{1}, \ldots, j_{n}, 1\right) \\
& \text { if } j_{0} \leq M, j_{t} \leq M_{t}, t=1, \ldots, n \\
= & -\infty \text { otherwise }
\end{aligned}
$$

Recurrence

$$
\begin{array}{r}
T\left(j_{0}, j_{1}, \ldots, j_{n}, i\right)=\max ( \\
T\left(j_{0}-l_{0}, j_{1}-l_{1}, \ldots, j_{n}-l_{n}, i-1\right)+z_{i}\left(l_{0}, l_{1}, \ldots, l_{n}\right): \\
l_{0} \in\left\{0,1, \ldots, \min \left(j_{0}, M\right)\right\}, l_{v} \in\left\{0,1, \ldots, \min \left(j_{v}, M_{v}\right)\right\}, \\
v=1, \ldots, n)
\end{array}
$$

It can be shown that $T\left(M, m_{1} M_{1}, \ldots m_{n} M_{n}, N\right)$ found from the above recurrence is the revenue of the feasible allocation $\left\{n_{i, j}: i=1, \ldots N ; j=0, \ldots, n\right\}$ that achieves the maximum revenue for the fixed values $M_{1}, \ldots, M_{n}$ assumed. Also, the revenue maximizing feasible allocation itself can be found from the array $T\left(j_{0}, j_{1}, \ldots j_{n}, i\right)$.

For all sets $M_{1}, \ldots, M_{n}$ such that $M_{1}+\ldots+M_{n}=M$, $T\left(M, m_{1} M_{1}, \ldots m_{n} M_{n}, N\right)$ and the revenue maximizing feasible allocation are found as explained above. Then the optimal set $\left(M_{1}^{*}, \ldots, M_{n}^{*}\right)$ is found as follows:

$$
\left(M_{1}^{*}, \ldots, M_{n}^{*}\right)=\underset{M_{1}+\ldots+M_{n}=M}{\operatorname{argmax}} T\left(M, m_{1} M_{1}, \ldots m_{n} M_{n}, N\right)
$$

The revenue maximizing feasible allocation with $M_{1}=M_{1}^{*}$, $\ldots, M_{n}=M_{n}^{*}$ is the one that maximizes revenue among all feasible allocations.

LEMMA 3. The running time of the above algorithm is $O\left(M^{3 n+2} m_{n}^{n} N\right)$.

LEMMA 4. The maximum amount of storage required at any given time during the execution of the algorithm is $O\left(M^{n+1} m_{n}^{n} N\right)$.

Note that the running time and space complexity of the algorithm are polynomial for fixed $n$.

\section{A GREEDY 2-APPROXIMATION ALGORITHM}

The scheme described in Section 5 is feasible for fixed $n$, the number of possible cardinalities of the set of secondary networks on a channel. However, if $n$ is allowed to grow, the set of bids of a network is exponential in size as Lemma 1 shows and hence the scheme is infeasible. In this section, we first provide a compact bidding language for the case with large $n$, i.e. a means for networks to approximately convey their bids. We conjecture that under this bidding language, the access allocation problem is NP-hard. We give a basis for this conjecture in Section 7. We provide a polynomial-time algorithm that approximates the maximum revenue of the auctioneer within a factor of 2 . Note that this algorithm can also be used to approximate the maximum social welfare of the bidding networks within a factor of 2 using the extended second-chance mechanism described in Section 3.

We describe the bidding language in Section 6.1. In Section 6.2, we introduce residual bid functions, a concept used in the approximation algorithm. We describe the algorithm in Section 6.3 and prove that it achieves an approximation ratio of 2 in Section 6.4.

\subsection{Bidding Language}

Consider the model in Section 5 with the following changes. Let the bandwidth of each of the $M$ channels be $W$ bps. We assume that the primary network on a channel uses the channel for an expected fraction of time $\alpha$, where $0<\alpha<1$. When auctions are repeated periodically to assign spectrum, $\alpha$ can be estimated based on long-term measurements of the primary networks' channel usage. Alternatively, it can be estimated via simulations. Since secondary networks can use the channel whenever the primary is not using it, an expected bandwidth of $W(1-\alpha)$ is available on a channel for the secondary networks. So when $m_{j}$ secondary networks 
share a channel, each one of them can get an expected secondary throughput of $\frac{W(1-\alpha)}{m_{j}}$ on the channel. In this section, we allow a network $i$ to express bids as a function of the number of channels $n_{i, 0}$ on which it is primary and its total expected secondary throughput $T_{i}^{s}$ on all $M$ channels. Note that:

$$
T_{i}^{s}=\sum_{j=1}^{n} \frac{n_{i, j} W(1-\alpha)}{m_{j}}
$$

In the sequel, for brevity, we simply say secondary throughput instead of expected secondary throughput. Moreover, we assume that the utility, and hence the bid $z_{i}\left(n_{i, 0}, T_{i}^{s}\right)$, of each network $i$ when it is primary on $n_{i, 0}$ channels and has $T_{i}^{s}$ units of secondary throughput, is separable, i.e. of the form:

$$
z_{i}\left(n_{i, 0}, T_{i}^{s}\right)=w_{i}\left(n_{i, 0}\right)+y_{i}\left(T_{i}^{s}\right)
$$

where $w_{i}\left(n_{i, 0}\right)$ is its bid for being primary on $n_{i, 0}$ channels and $y_{i}\left(T_{i}^{s}\right)$ is its bid for $T_{i}^{s}$ units of throughput as a secondary network. This assumption is a good approximation since networks transmit different kinds of traffic (delay-sensitive and elastic respectively) as a primary and secondary network.

Under this assumption, the access allocation problem separates out into two independent problems- allocating the primary parts and allocating the secondary parts. The problem of allocating the primary parts can be optimally solved in $O\left(M^{2} N\right)$ time using the dynamic programming algorithm in Section 5.2 with $n=0$. In this section, we focus on giving a 2-approximation algorithm for the problem of allocating the secondary parts so as to maximize the auctioneer's revenue. In the rest of the section, "revenue" refers to the auctioneer's revenue from selling the secondary rights on the $M$ channels.

Assume that $y_{i}($.$) is a concave increasing function for each$ network $i$. We use piecewise linear concave functions to compactly represent the bid functions of the networks. They can be used to closely approximate arbitrary concave functions [16] and have been previously used in the context of spectrum auctions in [9]. Each network $i$ specifies its bid for at most $P$ different levels of secondary throughput, for a positive integer $P$. More precisely, let $P_{i} \leq P$ be a positive integer and let:

$$
0=q_{i, 1}<q_{i, 2}<\ldots<q_{i, P_{i}}
$$

For $v=1, \ldots, P_{i}$, network $i$ specifies $y_{i}\left(q_{i, v}\right)$, which is its bid for $q_{i, v}$ units of secondary throughput. Network $i$ 's bid for $q$ units of secondary throughput, where $q_{i, v}<q<q_{i, v+1}$ is found by linear interpolation:

$$
y_{i}(q)=y_{i}\left(q_{i, v}\right)+\left(\frac{y_{i}\left(q_{i, v+1}\right)-y_{i}\left(q_{i, v}\right)}{q_{i, v+1}-q_{i, v}}\right)\left(q-q_{i, v}\right)
$$

We assume that for each network $i, q_{i, 1}=0$, that $y_{i}\left(q_{i, 1}\right)=$ $y_{i}(0)=0$ and that

$$
q_{i, P_{i}} \geq M W(1-\alpha) .
$$

Since $M W(1-\alpha)$ is the total secondary throughput available on the $M$ channels, the second assumption means that network $i$ 's bid for any amount of secondary throughput on the $M$ channels can be found by linear interpolation.

\subsection{Residual Bid Functions}

Our algorithm uses the following concept.
DeFinition 2. Let $\tilde{q} \geq 0$. The $\tilde{q}$-residual bid function of network $i$ is the function $\tilde{y}_{i}($.$) given by:$

$$
\tilde{y}_{i}(q)=y_{i}(\tilde{q}+q)-y_{i}(\tilde{q})
$$

We will sometimes say, "the residual bid function after accounting for $\tilde{q}$ " instead of the $\tilde{q}$-residual bid function. Informally, once network $i$ has been allocated $\tilde{q}$ units of secondary throughput, $\tilde{y}_{i}($.$) acts as its bid function for allocations of$ additional secondary throughput. It can be shown that the residual bid function can be efficiently computed from the bid function. We omit the proof due to space constraints. The following lemma gives some simple properties about the $\tilde{q}$-residual bid function.

Lemma 5. Let $\tilde{y}_{i}(q)$ be the $\tilde{q}$-residual bid function of network $i$ for some $\tilde{q} \geq 0$. Then

1. $\tilde{y}_{i}(q) \leq y_{i}(q) \forall q \geq 0$.

2. $\tilde{y}_{i}(q)$ is a concave increasing function.

The significance of the $\tilde{q}$-residual bid function is given by the following lemma.

LEMma 6. Suppose the bid function of network $i$ is $y_{i}($. and it is successively allocated secondary throughputs of $q_{1}, q_{2}, \ldots, q_{f}$. Let $y_{i}^{v}($.$) denote the \left(q_{1}+\ldots+q_{v}\right)$-residual bid function of network $i$, for $v=1, \ldots, f$. Then

$$
y_{i}\left(q_{1}+\ldots+q_{f}\right)=y_{i}\left(q_{1}\right)+y_{i}^{1}\left(q_{2}\right)+\ldots+y_{i}^{f-1}\left(q_{f}\right)
$$

Thus, the significance of the residual bid function is that if a network $i$ is successively allocated chunks $q_{1}, \ldots, q_{f}$ of secondary throughput (e.g. by successive steps of an algorithm), then we can keep track of its residual bid function after every allocation so that the extra money that network $i$ is willing to pay for the $v^{\prime}$ th allocation $q_{v}$ is simply $y_{i}^{v-1}\left(q_{v}\right)$. Moreover, this tracking can be done using the update rule in part 1 of the following lemma to calculate $y_{i}^{v+1}($.$) from$ $y_{i}^{v}($.$) .$

Lemma 7. Let $\tilde{y}_{i}($.$) and y_{i}^{+}($.$) be the \tilde{q}$-residual bid function and $(\tilde{q}+\hat{q})$-residual bid function of network $i$ respectively. Then

$$
\begin{aligned}
& \text { 1. } y_{i}^{+}(q)=\tilde{y}_{i}(q+\hat{q})-\tilde{y}_{i}(\hat{q}) \forall q \geq 0 \\
& \text { 2. } y_{i}^{+}(q) \leq \tilde{y}_{i}(q) \forall q \geq 0 .
\end{aligned}
$$

Note that $y_{i}^{+}($.$) is the \hat{q}$-residual bid function corresponding to the bid function $\tilde{y}_{i}($.$) .$

\subsection{Algorithm Description}

We now describe the 2-approximation algorithm. For each network $i$, the residual bid function $\tilde{y}_{i}($.$) is initialized to$ $y_{i}($.$) .$

The algorithm successively determines into how many secondary parts channel $l$ is to be divided and which networks are to be the secondary networks on that channel, for $l=$ $1, \ldots, M$, one channel at a time. Suppose we have taken these decisions for channels $1,2, \ldots, l-1$ and for each network $i$, have set $\tilde{y}_{i}($.$) to be equal to its residual bid function$ after accounting for the secondary throughput allocated to it in the first $l-1$ channels. Assign channel $l$ using the following steps:

STEP1: For $j=1, \ldots, n$, find the maximum increase in revenue $R_{j}^{l}$ obtainable from channel $l$ by dividing the channel 
into $m_{j}$ secondary parts using the following rule. Sort the set of numbers $\tilde{y}_{i}\left(\frac{W(1-\alpha)}{m_{j}}\right), i=1, \ldots, N$ into decreasing order. Let $\tilde{y}_{(v)}\left(\frac{W(1-\alpha)}{m_{j}}\right)$ denote the $v^{\prime}$ th largest element. Then $R_{j}^{l}$ is given by:

$$
R_{j}^{l}=\sum_{v=1}^{m_{j}} \tilde{y}_{(v)}\left(\frac{W(1-\alpha)}{m_{j}}\right)
$$

STEP2: Find the maximum among $R_{1}^{l}, \ldots, R_{n}^{l}$. Suppose $R_{j}^{l}$ is the maximum. Then divide the $l$ 'th channel into $m_{j}$ secondary parts. On the $l$ 'th channel, the $m_{j}$ networks with the $m_{j}$ largest values $\tilde{y}_{(1)}\left(\frac{W(1-\alpha)}{m_{j}}\right), \ldots, \tilde{y}_{\left(m_{j}\right)}\left(\frac{W(1-\alpha)}{m_{j}}\right)$ become secondary networks.

STEP3: Update the function $\tilde{y}_{i}($.$) of each one of the m_{j}$ networks that become the secondary networks on channel $l$ to its residual bid function after accounting for the secondary throughput allocated to it in the first $l$ channels.

\subsubsection{Comments on Algorithm}

Once channels $1, \ldots, l-1$ have been allocated, steps 1 and 2 allocate channel $l$ so as to obtain the maximum possible increase in revenue over the revenue from channels $1, \ldots, l-$ 1. In Step 3, the rule in part 1 of Lemma 7 can be used to update the residual bid functions of the networks who become secondary networks on channel $l$.

\subsection{Approximation Ratio}

TheOREM 3. Let $R^{*}$ be the maximum possible revenue under any allocation of the rights to be secondary networks on the $M$ channels and let $R^{G}$ be that achieved by the above greedy algorithm. Then $R^{G} \geq \frac{R^{*}}{2}$.

Proof. Let $R^{l}$ be the increase in revenue obtained by the greedy algorithm from allocating the l'th channel. Denote by $q_{i, l}^{G}$, the amount of secondary throughput allocated by the greedy algorithm to network $i$ in the $l$ 'th channel. Let $y_{i}^{l}($.$) be the \left(q_{i, 1}^{G}+\ldots+q_{i, l}^{G}\right)$-residual bid function of network $i$, that is, its residual bid function after accounting for the secondary throughput allocated to it in channels 1 to $l$. By part 2 of Lemma 7:

$$
y_{i}^{l}(q) \leq y_{i}^{l-1}(q) \forall q \geq 0
$$

From the discussion after Lemma 6, it follows that after channels $1, \ldots, l$ were allocated, the extra money network $i$ is willing to pay for its share in channel $(l+1)$ is $y_{i}^{l}\left(q_{i, l+1}^{G}\right)$. Moreover, if the greedy algorithm were to allocate the l'th channel to the same set of networks to whom it actually allocated the $(l+1)$ 'st channel, then (a) after channels $1, \ldots, l-1$ were allocated, the extra money network $i$ would be willing to pay for its share in channel $l$ would have been $y_{i}^{l-1}\left(q_{i, l+1}^{G}\right)$ and hence by (18), (b) the increase in revenue from the $l$ 'th channel would have been at least $R^{l+1}$. But the actual increase in revenue from the $l^{\prime}$ th channel, $R^{l}$, is by definition of the greedy rule, the maximum possible from allocating the l'th channel. Hence $R^{l} \geq R^{l+1}$. Thus, we get:

$$
R^{1} \geq R^{2} \geq \ldots \geq R^{M}
$$

Since $R^{G}=R^{1}+\ldots+R^{M}$, we get:

$$
R^{M} \leq \frac{R^{G}}{M}
$$

Now, let $q_{i}^{*}$ be the total secondary throughput allocated by the optimal algorithm to network $i$ and $q_{i}^{G}$ be that allocated by the greedy algorithm. Also, let $S_{l}^{*}$ be the set of secondary networks on the l'th channel, $l=1, \ldots, M$, in the optimal allocation. Next, we will upper bound $R^{*}-R^{G}$, the excess revenue of the optimal allocation over the greedy allocation. To this end, for each network $i$, we account for its payment for $\max \left(q_{i}^{*}-q_{i}^{G}, 0\right)$, the excess secondary throughput if any, of the optimal allocation over the greedy algorithm's allocation, by accounting for its payments for the chunks $q_{i, l}^{e}, l=1, \ldots, M$. Here, $q_{i, l}^{e}$ is the contribution of channel $l$ to the excess $\max \left(q_{i}^{*}-q_{i}^{G}, 0\right)$, once the contributions of channels $1, \ldots, l-1$ have been accounted for and is given by:

$$
\begin{array}{r}
q_{i, l}^{e}=\min \left(\frac{W(1-\alpha)}{\left|S_{l}^{*}\right|},\right. \\
\left.\max \left(q_{i}^{*}-q_{i}^{G}-q_{i, 1}^{e}-\ldots-q_{i, l-1}^{e}, 0\right)\right), \quad i \in S_{l}^{*} \\
q_{i, l}^{e}=0, \quad i \notin S_{l}^{*}
\end{array}
$$

We get the expression in (20) as follows. First, since channel $l$ is shared by $\left|S_{l}^{*}\right|$ networks, $q_{i, l}^{e} \leq \frac{W(1-\alpha)}{\left|S_{l}^{*}\right|}$. The second term in the min is equal to the as yet unaccounted for excess, if any, obtained by subtracting the contributions $q_{i, 1}^{e}, \ldots, q_{i, l-1}^{e}$ of channels $1, \ldots, l-1$ from the total excess throughput $\max \left(q_{i}^{*}-q_{i}^{G}, 0\right)$.

Let $y_{i, l}^{e}($.$) be the \left(q_{i}^{G}+q_{i, 1}^{e}+\ldots+q_{i, l}^{e}\right)$-residual bid function of network $i$. That is, $y_{i, l}^{e}($.$) is the residual bid function$ after accounting for the amount of secondary throughput allocated to network $i$ by the greedy algorithm $\left(q_{i}^{G}\right)$ and the contributions $q_{i, 1}^{e}, \ldots, q_{i, l}^{e}$ of the first $l$ channels to the excess $\max \left(q_{i}^{*}-q_{i}^{G}, 0\right)$.

Now,

$$
\begin{aligned}
R^{*} & -R^{G} \\
& \leq \sum_{l=1}^{M}\left(\sum_{i \in S_{l}} y_{i, l-1}^{e}\left(q_{i, l}^{e}\right)\right) \\
& \leq \sum_{l=1}^{M}\left(\sum_{i \in S_{l}} y_{i}^{M-1}\left(q_{i, l}^{e}\right)\right) \\
& \leq \sum_{l=1}^{M}\left(\sum_{i \in S_{l}} y_{i}^{M-1}\left(\frac{W(1-\alpha)}{\left|S_{l}^{*}\right|}\right)\right) \\
& \leq \sum_{l=1}^{M} R^{M} \\
& =M R^{M} \\
& \leq M\left(\frac{R^{G}}{M}\right) \\
& =R^{G}
\end{aligned}
$$

from which it follows that $R^{*} \leq 2 R^{G}$. We get (22) as follows. The excess revenue $R^{*}-R^{G}$ arises from the extra payment that each network $i$ makes for the excess secondary throughput $\max \left(q_{i}^{*}-q_{i}^{G}, 0\right)$, if any, that it receives under the optimal allocation over the greedy allocation. Expression (22) accounts for these extra payments by adding, for channels $l=1, \ldots, M$, the total payments (see the discussion after Lemma 6 ) of all networks $i \in S_{l}$ for the contri- 
butions $q_{i, l}^{e}$ of channel $l$ to the excess secondary throughput $\max \left(q_{i}^{*}-q_{i}^{G}, 0\right)$. There is an inequality instead of equality in (22) because $q_{i}^{G}$ may be greater than $q_{i}^{*}$ for some networks $i$. Inequality (23) follows by part 2 of Lemma 7 , since $y_{i}^{M-1}($.$) is the residual bid function of network i$ after accounting for the amount of secondary throughput allocated to it by the greedy algorithm in the first $M-1$ channels, whereas $y_{i, l-1}^{e}($.$) is the residual bid function after account-$ ing for the secondary throughput allocated to it in all $M$ channels as well as the contributions $q_{i, 1}^{e}, \ldots, q_{i, l-1}^{e}$ of the first $l-1$ channels to the excess $\max \left(q_{i}^{*}-q_{i}^{G}, 0\right)$. Inequality (24) follows from the fact that $q_{i, l}^{e} \leq \frac{W(1-\alpha)}{\left|S_{l}^{*}\right|}$ and since $y_{i}^{M-1}($.$) is increasing by part 2$ of Lemma 5 . In inequality (25), we use the fact that

$$
\sum_{i \in S_{l}} y_{i}^{M-1}\left(\frac{W(1-\alpha)}{\left|S_{l}^{*}\right|}\right) \leq R^{M}
$$

which is true because when the greedy algorithm was about to allocate channel $M$, the increase in revenue it would have got from the channel if it allocated the channel to the $\left|S_{l}^{*}\right|$ networks in the set $S_{l}^{*}$ is equal to the expression on the left hand side of (29). This expression is at most $R^{M}$, since the greedy algorithm allocates the $M$ 'th channel so as to maximize the increase in revenue from it. Finally, inequality (27) follows from (19).

LEMMA 8. The running time of the above greedy algorithm is $O\left(n M N \log (P N)+M P m_{n}\right)$.

\section{CONCLUSIONS AND FUTURE WORK}

We developed a comprehensive framework for access allocation in cognitive radio networks. We proposed three bidding languages, each less expressive than the previous one, but suitable for larger auctions. Also, we developed a number of algorithms for the access allocation problem for these bidding languages.

In Section 6, we gave a polynomial-time 2-approximation algorithm for the problem, described in Section 6.1, of allocating secondary rights so as to maximize the auctioneer's revenue. We conjecture that it is NP-hard to solve it optimally. Our conjecture is motivated by the facts that (a) the bid function of each network can be an arbitrary realvalued function satisfying the conditions in Section 6.1, (b) the number of secondary networks on each channel can be selected from a possibly large set $\left\{m_{1}, \ldots, m_{n}\right\}$ and (c) the set of secondary networks on each channel can be an arbitrary subset of the set of all $N$ networks. We leave the question of NP-hardness as an open problem for future research.

Also, we considered the case when the $M$ channels are identical. The extension to non-identical channels can be considered as part of future work.

\section{ACKNOWLEDGMENTS}

The contributions of both authors have been supported by NSF grants NCR-0238340, CNS-0721308, ECS-0622176.

\section{REFERENCES}

[1] Q. Zhao and B. Sadler "A Survey of Dynamic Spectrum Access". In IEEE Signal Processing Magazine, Vol. 24, Issue 3, pp. 79-89, 2007.
[2] FCC Spectrum Policy Task Force "Report of the Spectrum Efficiency Working Group". Nov. 2002. Available at: http://www.fcc.gov/sptf/reports.html

[3] FCC Auctions http://wireless.fcc.gov/auctions/

[4] I. Akyildiz, W.-Y. Lee, M. Vuran, S. Mohanty "NeXt generation/dynamic spectrum access/cognitive radio wireless networks: a survey". In Computer Networks, Vol. 50, Issue 13, pp. 2127-2159, 2006.

[5] D. Bertsekas, R. Gallager, "Data Networks". Prentice Hall, Englewood Cliffs, New Jersey, second edition, 1992.

[6] Z. Ji, K.J. Ray Liu, "Belief-Assisted Pricing for Dynamic Spectrum Allocation in Wireless Networks with Selfish Users". In Proc. of IEEE SECON, 2006.

[7] J. Huang, R. Berry, M. Honig "Auction Mechanisms for Distributed Spectrum Sharing". In Proc. of 42nd Allerton Conference, 2004.

[8] S. Sengupta, M. Chatterjee "Sequential and Concurrent Auction Mechanisms for Dynamic Spectrum Access". In Proc. of CROWNCOM, 2007.

[9] S. Gandhi, C. Buragohain, L. Cao, H. Zheng, S. Suri, "Towards Real-Time Dynamic Spectrum Auctions". In Computer Networks, Vol. 52, Issue 4, March 2008, Pp. 879-897.

[10] J. Peha, "Emerging Technology and Spectrum Policy Reform" In Proc. of ITU Workshop on Market Mechanisms for Spectrum Management, Jan. 2007.

[11] P. Cramton, Y. Shoham, R. Steinberg, "Combinatorial Auctions". MIT Press, 2006

[12] M. Tennenholtz, "Some Tractable Combinatorial Auctions". Proc. of National Conference on Artificial Intelligence (AAAI), 2000

[13] J. Edmonds "Maximum Matching and a Polyhedron with 0,1-Vertices". In Journal of Research of the National Bureau of Standards, 69B, 125-130, 1965.

[14] L. White "A Parametric Study of Matchings and Coverings in Weighted Graphs" Ph.D. Dissertation, University of Michigan, Ann Arbor, 1967.

[15] L. White "An Efficient Algorithm for Maximum k-Matching in Weighted Graphs". In Proc. of 12th Allerton Conference on Circuits and Systems Theory, 1974.

[16] D. Bertsimas and J. Tsitsiklis "Introduction to Linear Optimization". Athena-Scientific, 1997.

[17] A. Mas-Colell, M. Whinston and J. Green "Microeconomic Theory". Oxford University Press, 1995.

[18] J. Kleinberg and E. Tardos "Algorithm Design". Addison Wesley, 2005.

[19] N. Nisan and A. Ronen "Computationally Feasible VCG Mechanisms". In Proc. of ACM Conference on Electronic Commerce, 2000.

[20] A. Ronen "Mechanism Design with Incomplete Languages". In Proc. of ACM Conference on Electronic Commerce, 2001. 